\title{
Azoospermic patient's treatment: An experience of a PMA hospital unit and role of ultrasonography
}

\author{
Paolo Panella ${ }^{1}$, Pietro Pepe ${ }^{1}$, Placido Borzi ${ }^{2}$, Maria Elena Vento ${ }^{2}$, Michele Pennisi ${ }^{1}$, Paolo Scollo ${ }^{2}$ \\ ${ }^{1}$ Urologic Unit, ${ }^{2}$ Obstetrics and Gynecology Unit, PMA service, Cannizzaro Hospital, Catania, Italy.
}

\begin{abstract}
Summary Introduction: Azoospermia causes about $10 \%$ of male infertility and the best therapeutic option is the retrieval of sperm from testis or epididymis.

Material and methods: From Juanary 2008 to June 2016, 92 men (median 36 years; range: 25-54 years) were submitted in 47 cases to TESE (testicular sperm extraction) and in 45 cases to PESA (percutaneous epididymal sperm aspiration) for secretory and obstructive azoospermia, respectively; moreover, all the patients previously underwent color Doppler ultrasound of the testis and transrectal ultrasound of the prostate.

Results: Serum FSH values were $9.4 \mathrm{ml} / \mathrm{UI}$ and $36.4 \mathrm{ml} / \mathrm{UI}$ (median $18.2 \mathrm{ml} / \mathrm{UI}$ ) with an estimated volume of the testis equal to $5 \mathrm{ml} ; 40 \mathrm{men}$ had the mutation for cystic fibrosis with bilateral agenesis of the deferentia vasa, 4 men had a cyst of the prostatic utricle, 1 man had retrograde ejaculation, 7 had an epididymis cyst and 2 had anejaculation secondary to traumatic neurologic spinal cord injury. The retrieval of sperm was performed in 39 (83\%) and 36 (80\%) of the patients submitted to TESE and PESA, respectively. The pregnancy rate was equal to $28 \%$ and $33 \%$ in men with secretory and obstructive azoospermia, respectively. Discussion: Assisted reproduction technology with a multidisciplinary team is provided of a pregnancy rate equal about $30 \%$ in men with azoospermia; ultrasound allows to evaluate abnormalities of the testis and prostate improving the percentage of pregnancy.
\end{abstract}

KEY WORDS: Azoospermia; Sperm retrieval techniques; Ultrasound of male genital tract; Treatment of azoospermia.

Submitted 15 November 2016; Accepted 15 December 2016

\section{INTRODUCTION}

About $20 \%$ of the couples is unable to have a pregnancy. The obstructive and non obstructive azoospermia constitutes the $10 \%$ of male infertility; in these cases the ICSI (intracytoplasmatic sperm injiection) allows to have a pregnancy in about $30 \%$ of the cases (1). The sperm could be retrieval in the semen or directly in the testis or epididymis performing a percutaneous or open procedure (2-4). The role of color Doppler ultrasound (CDU) in the clinical evaluation of azoospermic men, has been reported (5).

\section{MATERIAL AND METHODS}

From January 2008 to June 2016, 92 men (median 36 years; range: $25-54$ years) were submitted in 47 cases to
TESE (testicular sperm extraction) and in 45 cases to PESA (percutaneous epididymal sperm aspiration) for secretory and obstructive azoospermia, respectively; moreover, all the patients previously underwent CDU of the testis and prostate. The PESA was performed under local anesthesia (mepivacaine 2\% without adrenaline) using a 21 gauge butterfly. All the patients underwent to general and genital physical examination, evaluation of two semen analysis, culture of semen performing PCR (amplification of DNA) for Chlamidya trachomatis, Mycoplasma hominis and mycetes. In addition, the karyotype with the evaluation of microdeletion for cromosoma Y and mutation of fibrosis cistyc genes was done. A GE Logiq E9 and P6 with small part and biplanar transrectal probes were used to perform CDU evaluation of testis and prostate; in detail, the ecopattern and the vessels maps of the testis were evaluated to improve the detection of sperm during biopsy procedure. All the patients underwent to blood evaluation for $\mathrm{FSH}, \mathrm{LH}$, testosterone, CMV, HBV, HCV e HIV. The biopsy testicular parenchyma was delivered to the biologists team to retrieval the sperm and perform in real time ICSI procedure; finally, testicular parenchyma underwent definitive histological evaluation.

\section{RESULTS}

Serum FSH values were $9.4 \mathrm{ml} / \mathrm{UI}$ and $36.4 \mathrm{ml} / \mathrm{UI}$ (median $18.2 \mathrm{ml} / \mathrm{UI}$ ) with an estimated volume of the testis equal to $5 \mathrm{ml} ; 40$ men had the mutation for cystic fibrosis with bilateral agenesis of the deferentia vasa, 4 men had a cyst of the prostatic utricle, 1 man had retrograde ejaculation, 7 had and epididymis cyst and 2 had anejaculation secondary to traumatic neurologic spinal cord injury. The recovery of sperm was performed in 39 $(83 \%)$ and $36(80 \%)$ of the patients submitted, respectively, to TESE and PESA. The pregnancy rate was equal to $28 \%$ in men with secretory azoospermia and to $33 \%$ in those with obstructive azoospermia. The culture of semen was negative in all patients. Four men underwent transperineal ultrasound guided prostatic cyst aspiration before testis biopsy (Figure 1); none had abnormal ultrasound suspicious area of the testis. Three patients had hematocele and orchiepididymitis following biopsy those did not required hospitalization. Definitive specimens did not found neoplastic lesions of the testis. 
Figure 1.

Transperineal percutaneous aspiration of prostatic cyst.
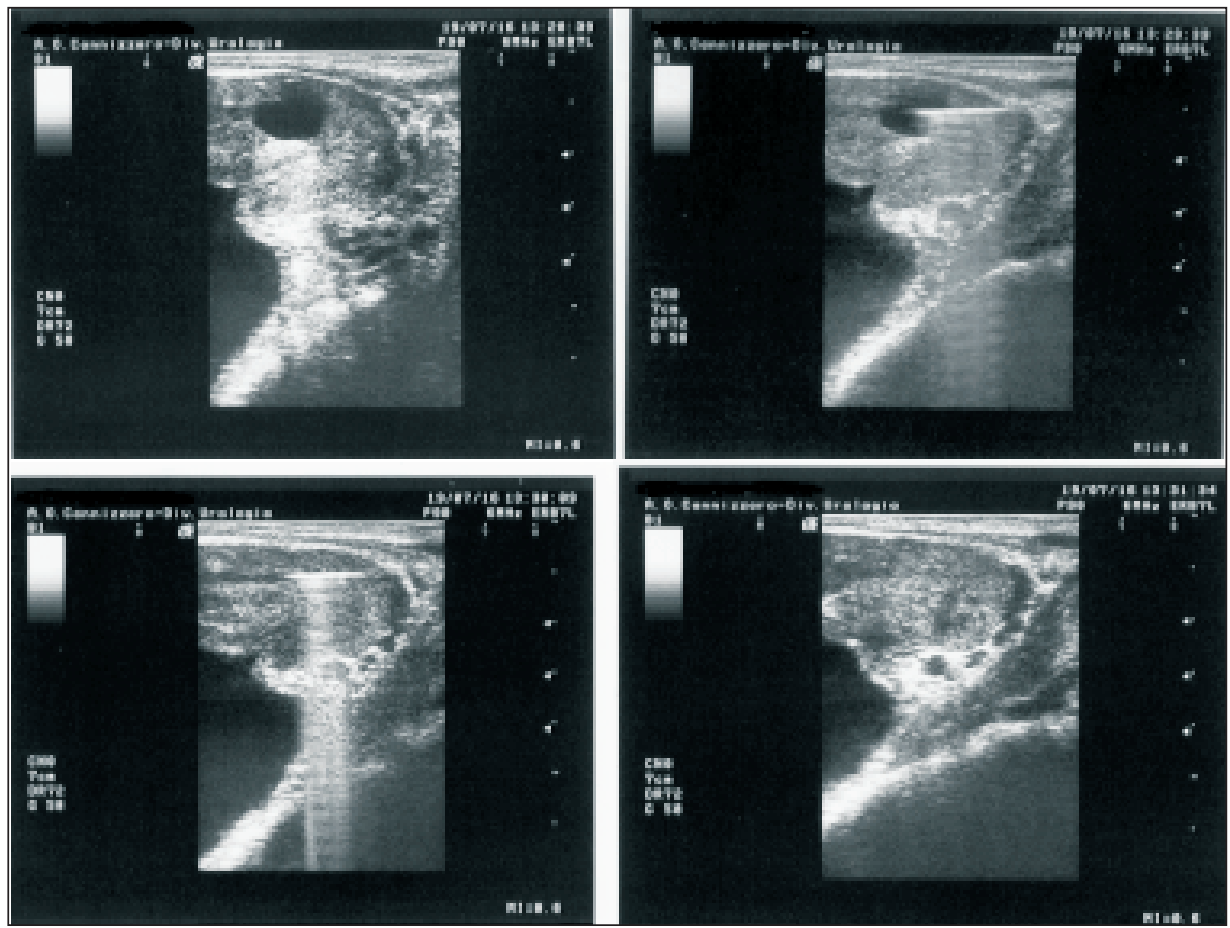

from the testicle, under local anesthesia, and extracting the few viable sperm cells present in that tissue for ICSI (Figure 2). MESA refers to retrieval of sperm-containing fluid from optimal areas of the epididymis that are selected and sampled using highpower optical magnification provided by an operating microscope (14).

The percutaneous approach is less invasive then surgical techniques and could be performed under local anesthesia; TESE and microTESE procedures allows to reach a sperm retrieval rates equal to $30-70 \%$ and $80 \%$ per cycle, respectively. In our series, we obtained a sperm retrieval rate equal to $78 \%$ with a pregnancy rate of 28\% (15). In conclusion, assisted reproductive department needs a multidisciplinary team to

\section{Discussion}

Assisted reproduction technology (ART) is the treatment used to achieve pregnancy in procedures such as fertility medication, artificial insemination, in vitro fertilization and surrogacy. It mainly belongs to the field of reproductive endocrinology and infertility, and may also ICSI and cryopreservation $(6,7)$. Some forms of ART are also used with regard to fertile couples for genetic reasons (preimplantation genetic diagnosis). Transvaginal ovum retrieval is the process whereby a small needle is inserted through the back of the vagina and guided via ultrasound into the ovarian follicles to collect the fluid that contains the egg cells. The ICSI is beneficial in the case of male factor infertility where sperm counts are very low, in the presence of azoospermia or failed fertilization occurred with previous IVF attempt. The ICSI procedure involves a single sperm carefully injected into the center of an egg cell using a microneedle $(8,9)$. Before performing ART clinical evaluation of the couple is mandatory; in this respect, CDU of the testis and prostate improve sperm retrieval rate guiding testis biopsy in the areas with better parenchymal microcirculation $(10,11)$. In addition, transrectal ultrasound detect seminal obstruction secondary to cistys or stenosis of ejaculatory ducts those could be successfully cured $(12,13)$.

The PESA is a technique used to determine sperm counts in the event of a possible blockage of the deferentia vasa; it is an alternative to microepidydimal sperm aspiration (MESA), and aims to address the technical difficulty and cost of MESA. A small needle (butterfly 23 o 21 G) is inserted through the skin of the scrotum to collect sperm from the epididymis or testis (TeFNA "testicular fine needle aspiration - TESA "testicular sperm aspiration"). The TESE is the process of removing a small portion of tissue improve the clinical couple evaluation and the pregnancy rate; CDU of testis and prostate allows to improve the percentage of pregnancy.

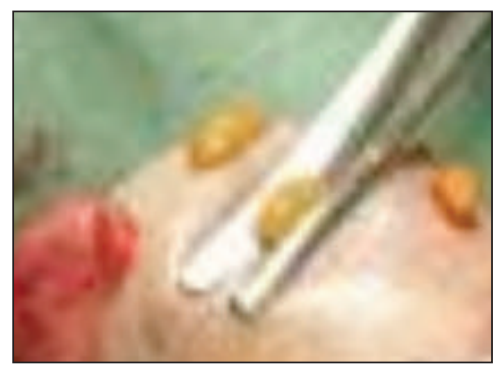

Figure 2.

Testicular sperm extraction.

\section{REFERENCES}

1. Timpano M, Fontana D, Rolle L, et al. Prelievo di spermatozoi per la procreazione medicalmente assistita in pazienti affetti da azoospermia. Urologia 2014; 81:27-31.

2. Esteves SC, Miyaoka R, Agarwal A. Sperm retrieval techniques for assisted reproduction. Int Braz J Urol. 2011; 37:570-583.

3. Colpi GM, Piediferro G, Nerva F, et al. Sperm retrieval for intracytoplasmic sperm injection in nonobstructive azoospermia. Minerva Urol Nefrol. 2005; 57: 99-107.

4. Tsujimura A, Matsumiya K, Koga M, et al. Outcome of surgical treatment for obstructive azoospermia. Arch Androl. 2002; 48:2936.

5. Lotti F, Maggi M. Ultrasound of the male genital tract in relation to male reproductive health. Hum Reprod Update. 2015; 21:56-83.

6. Hong-Tao Jiang, Qian Yuan, Yu Liu, et al. Multiple advance surgical techniques to treat acquired seminal duct obstruction. Asian J Androl. 2014; 16:912-916. 
7. Tsujimura A. Microdissection testicular sperm extraction: Prediction, outcome and complications. Int J Urol. 2007; 14:883889.

8. Schroeder-Printzen I, Ludwig M, Köhn F, Weidner W. Surgical therapy in infertile men with ejaculatory duct obstruction: technique and outcome of a standardized surgical approach. Hum Reprod. 2000; 15:1364-1368.

9. Watkins W, Nieto E, Bourne H, et al. Testicular and epididymal sperm in a microinjection program: methods of retrieval and results. Fertil Steril. 1997; 67:527-535.

10. Colpi GM, Colpi EM, Piediferro G, et al. Microsurgical TESE versus conventional TESE for ICSI in non-obstructive azoospermia: a randomized controlled study. Reproductive BioMedicine Online. 2009; 3:315-319.

11. Glander HJ, Horn LC, Dorschner W, et al. Probability to retrieve testicular spermatozoa in azoospermic patients. Asian J Androl. 2000; 2:199-205.

12. Cytron S, Avrech OM, Pinkas H, et al. Preliminary experience with ultrasound-guided percutaneous perineal aspiration of spermatozoa for ICSI in azoospermic patients with obstructed ejaculatory ducts. Hum. Reprod. 1996; 11 (Suppl. 1), Abstract T069.

13. Xu Chen, Hua Wang, Rong Pei Wu, et al. The performance of transrectal ultrasound in the diagnosis of seminal vesicle defects: a comparison with magnetic resonance imaging. Asian J Androl. 2014; 16:907-911

14. Safran A, Reubinoff BE, Porat-Katz A, et al. Assisted reproduction for the treatment of azoospermia. Hum Reprod. 1998; 13:4.

15. Heshmat S, Lo KC. Evaluation and treatment of ejaculatory duct obstruction in infertile men. Can J Urol. 2006; 13:18-21.

\section{Correspondence}

Paolo Panella, MD (Corresponding Author)

p.panella@alice.it

Pietro Pepe, MD

Michele Pennisi, MD

Urologic Unit, Cannizzaro Hospital, Catania, Italy

Placido Borzì, MD

Maria Elena Vento, MD

Paolo Scollo, MD

Obstetrics and Gynecology Unit - PMA service, Cannizzaro Hospital, Catania, Italy 